\title{
Associating sport skills through virtual games: an introductory approach
}

\begin{abstract}
According to NORMA ${ }^{\mathrm{TM}}$ Engaging Multimedia Design (NEMD)Model, engagement is determined by levels. The model suggests that anything could be learned, mastered and acquired when a person reach a high level of engagement while interacting with a multimedia interface. An experimental study was conducted to prove whether this claim is true. A sport virtual game was chosen as a vehicle for this study. The chosen sport game must be one that is unfamiliar to the selected audience; in this case, children to ensure that the skills gained were because of the fact of engagement and not because of the influence of previous knowledge about the game. The purpose was to see whether playing an engaging sports game virtually, even though the game is an unfamiliar one, could influence children in acquiring sports skills when playing it in the physical world. The chosen sport game was Rugby, a not widely known sport to children aged 9-10 years old in Malaysia's Primary School Education. The game is only introduced in Secondary Schools. In NEMD model, a game is engaging if the game can sustain users' attention for a period of $2 \mathrm{~h}$ and more. There are two forms of data gathered. One, the recorded facial expressions and gestures of subjects to prove that the game is very engaging and another, the screenshots of skills of recorded performance in two contrasting spaces, virtual and real. In the virtual space, the children played the virtual game for a period of $2 \mathrm{~h}$. In real-life space, the children played the actual game in the playing field. Data gathered have proven that an engaging virtual game could, not only, engaged children in playing the game but also assist them to acquire sports skills taught in real-life situations at a fast speed. The children were able to associate sports skills in Rugby such as scrum, swing, punt, throw-in, drop kick, tackling and catching when playing in the field. The game gave them a holistic view of the game thus enable them to grab meanings of these movements when introduced to the game much faster than the traditional.
\end{abstract}

Keyword: Sports skills; Sports games; Games; Engaging; Virtual games; Computer games; Teaching materials; Teaching methods; Metacognition 